\title{
Erratum to: Color-secure digital image compression
}

\author{
Tamer Rabie ${ }^{1}$
}

Published online: 17 October 2016

(C) Springer Science+Business Media New York 2016

\section{Erratum to: Multimed Tools Appl \\ DOI 10.1007/s11042-016-3942-9}

Due to an error during typesetting, the data " $\{0, \pi\}$ " found under the second paragraph of Section 3.2 was incorrectly written as " $\{0, \mathrm{n}\}$." The correct sentence should read as "Bracamonte et al. in [3,4] show that the DCT-phase, in spite of its binary values $\{0, \pi\}$, conveys significant amounts of information about its associated image."

The online version of the original article can be found at http://dx.doi.org/10.1007/s11042-016-3942-9.

Tamer Rabie

trabie@sharjah.ac.ae

1 Department of Electrical and Computer Engineering, University of Sharjah, Sharjah, United Arab Emirates 